\title{
A Multiresolution Markovian Fusion Model for the Color Visualization of Hyperspectral Images
}

\author{
Max Mignotte
}

\begin{abstract}
In this paper, we present a nonstationary Markov random field (MRF) fusion model for the color display of hyperspectral images. The proposed fusion or dimensionality reduction model is derived from the preservation of spectral distance criterion. This quantitative metric of good dimensionality reduction and meaningful visualization allows us to derive an appealing fusion model of high-dimensional spectral data, expressed as a Gibbs distribution or a nonstationary MRF model defined on a complete graph. In this framework, we propose a computationally efficient coarse-to-fine conjugate-gradient optimization method to minimize the cost function related to this energy-based fusion model. The experiments reported in this paper demonstrate that the proposed visualization method is efficient (in terms of preservation of spectral distances and discriminality of pixels with different spectral signatures) and performs well compared to the best existing state-of-the-art multidimensional imagery color display methods recently proposed in the literature.
\end{abstract}

Index Terms-Color display, complete graph, conjugategradient method, multidimensional imagery, multiresolution optimization, nonlocal Markov model, nonstationary Markov random field (MRF), visualization of hyperspectral images.

\section{INTRODUCTION}

$\mathbf{N}$ OWADAYS, hyperspectral imaging sensors are able to collect information from across the electromagnetic spectrum (from the ultraviolet to infrared) and to acquire several image data, of the same scene, simultaneously in many narrow and adjacent (progressively longer wavelength) spectral bands. The resulting (high spectral resolution) 3-D image, also called a hyperspectral data cube, makes it possible to derive, for each pixel, a continuous and unique reflectance spectrum which is of great importance in many applications. To name a few, this spectral data information is exploited in geological applications (for identifying Earth's surface materials, such as particular mineral deposits or type of vegetation), environmental applications (early-warning monitoring of water supplies), and military intelligence applications (the detection/classification issue of man-made objects and possibly hidden targets).

In practical applications, it may be interesting if this huge amount of high-dimensional spectral data information is reduced to three dimensions. This allows us to quickly display

Manuscript received December 3, 2009; revised March 4, 2010 and April 28, 2010. Date of publication August 5, 2010; date of current version November 24, 2010. This work was supported by the Natural Sciences and Engineering Research Council of Canada under an individual research grant.

The author is with the Département d'Informatique et de Recherche Opérationnelle, Faculté des Arts et des Sciences, Université de Montréal, Montréal, QC H3C 3J7, Canada (e-mail: mignotte@iro.umontreal.ca).

Color versions of one or more of the figures in this paper are available online at http://ieeexplore.ieee.org.

Digital Object Identifier 10.1109/TGRS.2010.2051553 this hyperspectral data cube into an informative color image (with red, green, and blue (RGB) channels) with a standard tristimulus device. The resulting (three-band) RGB color image provides a quick overview of existing materials and their distribution in the image scene and a rough determination of regions of interest and important features or man-made objects in the image (photointerpretation) for further analysis. Obviously, such a three-color channel display results in significant loss of information. Consequently, the main objective of this dimensionality-reduction-based visualization step consists in preserving as much information as possible, while maximizing, in a statistical criterion sense, the separability of each observed existing material (or class) in the final visualized color image. This allows us to display, for example, the different scene elements as distinctively as possible, in order to first perform a rough identification before exploiting any more powerful and time-consuming processing strategies, such as a segmentation or a classification method.

To this end, linear projection methods, such as independent component analysis (ICA) [1] and principal component (PC) analysis (PCA) [2], have commonly been proposed in the literature to obtain the first three principal R, G, and B image components to be finally visualized. However, since ICA and PCA are linear projection methods, both assume that the underlying data manifold is linear, which is not necessarily true in the case of hyperspectral images. Moreover, ICA is based on the assumption of mutually independent sources, which is not really the case of hyperspectral data [3], and since the classical PCA uses variance (from both image and noise, including interference) as the ranking criterion, PCs may not be ranked in terms of image content alone [4]. To overcome this limitation, a noise-adjusted PCA (NAPCA) [5] and its variant, namely, interference NAPCA algorithms [6], were proposed to more informatively rank PCs in terms of signal-to-interference-plusnoise ratio. In the same way, the maximum autocorrelation factor (MAF) analysis [7] was proposed to also rank PCs in terms of image content alone (without noise). The MAF transformation is a linear multivariate transformation (based on PCA) into new orthogonal variables that are ordered by decreasing autocorrelation. In this way, noise channels (with low autocorrelation) or noise components induced by electronic or aircraft engine can be identified and eliminated [8]. A recent and adaptive linear fusion technique, which solves this same problem, is also proposed in [9]. The goal of this technique is to maximize the mutual information between the original hyperspectral bands and the fused R, G, and B channels. Another interesting linear projection-based color visualization approach is proposed in [10] and further explored and discussed 
in [11]. In their approach, inspired by the human visual system (which converts broadband visible radiation into three signals roughly corresponding to $\mathrm{R}, \mathrm{G}$, and $\mathrm{B}$ channels), the three displayed channels are simply estimated by linear integrations of the original hyperspectral image weighted by three different and fixed spectral weighting envelopes, just like the human photopic (daylight) vision works. However, since the spectral weighting envelopes are fixed, there is no adaptation to specific image information.

Multiresolution methods based on pyramidal decompositions [12] and wavelet transforms [13] have also been reported. In these methods, selection and fusion rules determine which spectral band is most relevant in a neighborhood of a given pixel, and how the features of this selected band are then incorporated into the fused image. These methods implicitly assume that there is only one dominant band at each pixel, which is not true in multispectral imagery due to the existing large interband correlation.

In order to overcome the main limitation of these established linear projection methods which mainly do not consider the nonlinear characteristics of the hyperspectral data (whose multiple sources of nonlinearity have been pointed out in [14]), an alternative idea consists of exploiting a nonlinear method for dimensionality reduction into three bands, such as the locally linear embedding method [15]. Nevertheless, this technique is very time consuming compared to linear projection methods.

Let us also mention the low-complexity color display approach (suitable for hardware implementation) proposed in [16], which uses the 1-b transform of hyperspectral image bands for selecting three suitable bands for the RGB display, or the multivariate visualization technique proposed in [17], which uses a double color layers displaying simultaneously on the first layer (referred to as the background layer) the distribution of materials existing in the image scene and, in the second layer (detail layer), their respective composition (i.e., the socalled end-member materials) at the subpixel level. A recent paper in which different color display techniques are reviewed, compared, and evaluated is proposed in [18].

The visualization approach and the fusion model, presented in this paper, are derived from the now well-known preservation of spectral distance criterion which measures the agreement between the distance of spectrums associated to each pair of pixels and their perceptual color distance in the final fused image to be displayed. This intuitive criterion was already used in [19] for estimating an informative color mapping, allowing the efficient visualization of hyperspectral images. Using this criterion, the authors chose the strategy of transforming the initial nonlinear optimization problem into a linear algebra problem of matrix factorization or eigendecomposition. More precisely, they chose to decompose this 3-D mapping problem in two steps: a 2-D projection as a partial solution using classical PCA and a linear programming method to solve for 1-D coordinate in the third dimension. In their framework, their method remains mainly linear. Moreover, this two-step dimensionality reduction method closely depends on a preprocessing step which first uses a classical PCA to a few bands (20 in their application) and an initial segmentation using a nonoptimal median-cut algorithm. Another strategy is the one proposed in this paper. We will show that the resulting objective function to be optimized can be viewed as a Gibbs energy, related to a nonstationary Markov random field (MRF) model defined on a complete graph. In this context, there are efficient multiresolution optimization strategies which have been recently proposed and can be adapted to quickly and simply minimize the underlying Markovian energy function to this Markovian model and to directly estimate the optimal color mapping associated to a hyperspectral cube.

It must be noted that multiresolution optimization approaches, as multigrid techniques (the latter being used in [20] for the segmentation of hyperspectral images) belong to the so-called coarse-to-fine algorithm class. The general idea of coarse-to-fine methods is to construct approximate coarser versions of the (e.g., optimization) problem and to use the solution of the coarser (and, hence, computationally simpler) version of this problem to obtain a good initial guess that guides and accelerates the solution of finer versions. In multiresolution procedures, coarser versions of the original problem is simply view at multiple resolution levels and, consequently, the problems solved at coarser scales represent an approximation to the original problem, whereas in multigrid methods, the problems that are solved at coarser scales correspond exactly to the original problems but with a constrained set of allowed reconstruction [21], [22].

The remainder of this paper is organized as follows: Section II describes the proposed Markovian dimensionality reduction model. Section III describes the optimization strategy used to minimize the Gibbs energy field related to this fusion model. Finally, Sections IV and V present the quantitative metrics used to validate our algorithm and a set of experimental results and comparisons with existing multidimensional visualization techniques, respectively.

\section{Proposed Fusion Model}

\section{A. Distance-Preservation-Criterion-Based Fusion Model}

Let us define our terms. We consider a hyperspectral image as a cube or a 3-D array of observed pixels, made up of several 2-D arrays. We define an image by $I$ or $I(s, k)$, where $s$ indicates the spatial location and $k$ indexes the particular spectral band in which the pixel lies in the hyperspectral cube. We will use the term image slice to denote a 2-D array for a given $k$, i.e., $I(., k)$, where $N$ is the number of pixels in each image slice. We will use the term spectral vector to denote all pixels associated with a site $s$, i.e., $I(s,.) \in R^{K}$, where $K$ is the number of spectral bands.

In order to provide perceptually meaningful visualization, the main goal of our color visualization strategy is to preserve, as much as possible, and as in [19], the distance between the spectral vectors of each pair of pixels and their final perceptual color distance in the final displayed color image. As perceptual color space, we use the classical CIE $1976 L^{*}, a^{*}, b^{*}$ (LAB) color space which is approximately perceptually uniform (i.e., a color space in which the same amount in a color value produces a change of about the same visual importance). To evaluate the reliability of our algorithm in this distance preservation criterion, we will also use, in the following, the correlation-based metric proposed in [10] (see Section IV). 
In addition, herein, and contrary to [19], the aforementioned "Distance Preservation" criterion will be ensured for each of the three (predefined) subsets covering the overall available wavelengths of the original hyperspectral image. Each subset is associated to the three L, A, and B channels in this perceptual color space. The visualization technique we propose then seeks to find the three (LAB) bands that minimize (according to the "preservation of distances") the following set of objective functions:

$$
\left\{\begin{array}{l}
\hat{L}=\arg \min _{L} \sum_{s, t_{s \neq t}}\left(\beta_{s, t}^{\left[1: \frac{1}{3} K\right]}-\left(L_{s}-L_{t}\right)^{2}\right)^{2} \\
\hat{A}=\arg \min _{A} \sum_{s, t_{s \neq t}}\left(\beta_{s, t}^{]^{\frac{1}{3}} K: \frac{2}{3} K\right]}-\left(A_{s}-A_{t}\right)^{2}\right)^{2} \\
\hat{B}=\arg \min _{B} \sum_{s, t_{s \neq t}}\left(\beta_{s, t}^{]^{\frac{2}{3}} K: K\right]}-\left(B_{s}-B_{t}\right)^{2}\right)^{2}
\end{array}\right.
$$

where $\beta_{s, t}^{\left[k_{0}-k_{1}\right]}$ denotes the squared Euclidean distance associated between two spectral vectors, associated with the pair of sites or spatial locations $(s, t)$, and between the spectral bands $k_{0}$ and $k_{1} . L_{s}, A_{s}$, and $B_{s}$ denote the $L, B$, and $A$ components at site (or pixel) $s$, respectively. The summation $\sum_{s, t_{s \neq t}}$ is over all the pair of sites (i.e., for all sites $s$ and for all the pair of sites including $s$ ) existing in the $L, A$, and $B$ color bands of the final image to be displayed, respectively. In our model, the three predefined subsets designed to map the overall available wavelengths of the hyperspectral cube can be viewed as three nonoverlapping (equally spaced) rectangular spectral weighting windows [10].

It is worth noting that, in the proposed dimensionality reduction model [see (1)], the luminance, which is somewhat the most important piece of information in a visual context and/or the most important aspect of color choice, corresponds to the nonlinear reduction of the (most) visible reflectance spectra acquired by the Airborne Visible/Infrared Imaging Spectrometer (AVIRIS) hyperspectral imaging sensor (band number $\epsilon$ $[1:(1 / 3) K] \Leftrightarrow$ wavelength $\in[400 \mathrm{~nm}: 1100 \mathrm{~nm}])$. A component then corresponds to the next most visible spectra and infrared red (band number $\in](1 / 3) K:(2 / 3) K] \Leftrightarrow$ wavelength $\in] 1100 \mathrm{~nm}: 1800 \mathrm{~nm}]$ ) and, finally, $B$ component to the least (nonvisible) informative spectra (middle infrared). Experimentally speaking, this also (empirically) corresponds to the optimal division of the hyperspectral data cube, i.e., the optimal subsets that also ensure the best distance preservation score.

\section{B. Nonstationary Gibbs Energy Field}

Each one of the three objective functions to be minimized [see (1)] can be viewed as a Gibbs energy field related to a nonstationary (and nonlocal) MRF model defined on a complete graph ${ }^{1}$ with long-range pairwise interactions, i.e., binary cliques $\langle s, t\rangle$ (or pairwise of pixels). Each binary clique of this MRF model is associated to a nonstationary potential since this model is spatially variant and depends on the distance between the spectral vectors associated to each pair of pixels $(s, t)$.

\footnotetext{
${ }^{1}$ Each node of this graph or pixel $s$ is connected to all other pixels of the image.
}

In this Markovian framework, efficient optimization strategies have been recently studied and can be adapted to efficiently and quickly minimize this underlying Markovian energy function whose global minima is one band $(\hat{L}, \hat{A}$, or $\hat{B})$ of the image in the perceptual color space.

To this end, we can use a global optimization procedure such as a simulated annealing (SA) algorithm [23] or another stochastic optimizer [24] whose advantages are twofold: First, it has the capability of avoiding local minima, and second, it does not require a good initial guess in order to estimate the solution. An alternative approach to these stochastic (and computationally expensive) procedures is to use a classical gradient descent algorithm or a deterministic variant of the SA procedure, i.e., the iterative conditional modes introduced by Besag [25]. This method, which is simply a gradient descent alternating the directions, i.e., which selects a variable while keeping all other variables fixed, is deterministic and simple (it does not require an analytical expression of the derivative of the energy function to be optimized). Nevertheless, it suffers from the same disadvantage as the classical gradient: It requires a proper initialization of the image to be recovered (close to the optimal solution). Otherwise, it will converge toward a bad local minima. In order to solve this problem, we could take as initialization, i.e., for the first iteration of the gradient descent procedure (related, for example, to the search of the optimal L-band), the image candidate $\hat{L}$ ensuring, among the bands between the interval $\left[k_{0}-k_{1}\right]$, the minimal energy of the Gibbs energy function of our fusion model [see (1)].

Remember also that each of the objective function of our fusion model is of the form $\sum_{s, t_{s \neq t}} V_{s, t}($.$) with a summation$ over potential functions $V_{s, t}($.$) involving long-range pairwise$ pixel interactions and not only adjacent pixels, as it is the case in a classical first-order MRF. Moreover, the potential functions $V_{s, t}($.$) , involved in our optimization problem, are not metric or$ semimetric (since, in our case, $L_{s}=L_{t} \nLeftarrow V_{s, t}\left(L_{s}, L_{t}\right)=0$ ). Consequently, (1) does not belong to the class of energy functions that can be minimized via graph cut techniques, such as the expansion-move and swap-move algorithms (see [26, Sec. 1]). This is particularly true because $V_{s, t}($.$) clearly also does not$ satisfy the (necessary) condition of regularity (see [27, Sec. 4]), which is more relaxed applicability conditions required to get a graph representability for the energy minimization by graph cuts. Optimization algorithms, such as loopy belief propagation (LBP) [28], [29] or its variant, which is the so-called treereweighted message-passing algorithm [30], are more general and can be applied to any type of potential functions $V_{s, t}($.$) .$ However, in our case, these optimization techniques would be very slow and expensive in terms of memory requirement. In these optimization strategies, each node sends a (different) message (in fact, an integer) to each of its neighbors and receives a message from each neighbor (at each iteration). For our MRF model defined on a complete graph, there would be $O\left(N^{2}\right)$ messages (to compute and store) per iteration (with $N$ as the number of pixels of each mapping). Moreover, as the standard way of computing the messages is to explicitly minimize over $L_{s}$ for each choice of $L_{t}$ [29], it would take $O\left(k^{2}\right)$ time to compute each message with $k=256$, i.e., the number of existing discrete (LAB) color channel values. The 


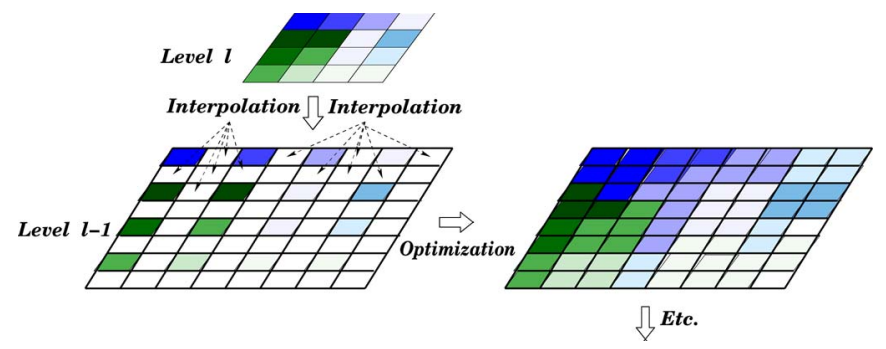

Fig. 1. Interpolation and "coarse-to-fine" minimization strategy.

standard implementation of these message-passing algorithms, on our (complete) graph, would thus require a prohibitive computational complexity of order $O\left(N^{2} k^{2} T\right)$ with $T \approx N^{1 / 2}$ (the number of iterations of the LBP needs to grow like $N^{1 / 2}$ [29] to allow for information from one part of the image to propagate everywhere else). In addition to this, LBP is not guaranteed to find a global minimum, only a strong local minimum [31]. Moreover, LBP is not also guaranteed to converge since it may go into an infinite loop switching between two labelings [31]. Note also that we cannot consider a dynamic programming approach [32] since this optimization method is restricted essentially to energy functions in 1-D settings.

Another robust optimization method of such a Gibbs energy field consists of a multiresolution approach combined with a gradient-descent-based optimization procedure. In this strategy, rather than considering the minimization problem on the full and original configuration space, the original optimization problem is decomposed in a sequence of approximated optimization problems of reduced complexity. This drastically reduces computational effort and provides an accelerated convergence toward improved estimate (experimentally, estimation results are nearly comparable to those obtained by stochastic optimization procedures as noticed, for example, in [33]-[35]) and in the following experimental results of this paper.

\section{COARSE-TO-Fine Optimization StRATEGY}

\section{A. Multiresolution Minimization Strategy}

To this end, we consider a multiresolution pyramid of image solutions at different resolution levels $l$ (resulting from the downsampling of $\hat{L}^{[0]} \equiv \hat{L}$ by $2^{\ell}$ in each direction) and a set of similar fusion models for each resolution level of this pyramidal data structure. For the search of the optimal L-band, at the upper level of the pyramidal structure (lower resolution level), the gradient descent optimization procedure is initialized with the image candidate $\hat{L}^{[l]}$ which ensures (among the bands between the interval $\left[k_{0}-k_{1}\right]$ ) the minimal energy of the Gibbs energy function of our fusion model [see (1.a)]. Since an analytical expression of the derivative of this energy function is easily available and in order to further speed up the optimization procedure at each level of the pyramidal structure, we use a conjugate-gradient procedure. After convergence of this procedure, the result obtained at this resolution level is interpolated and then used as initialization for the next finer level and so on, until the full resolution level (see Fig. 1).

For the interpolation at the next finer level, we have used an interpolation method which first takes efficiently into account
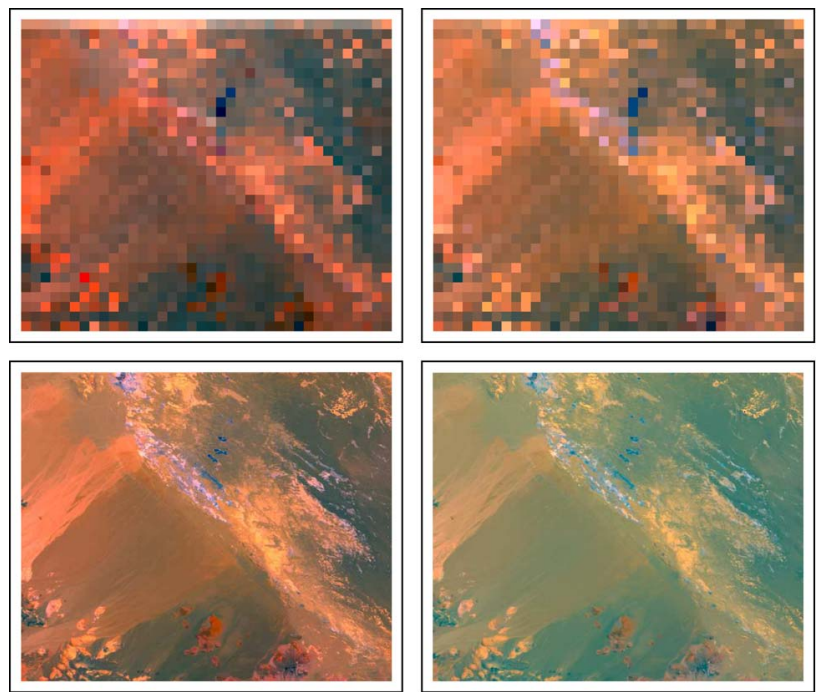

Fig. 2. Multiresolution optimization strategy on LunAR-LAKE $\mathrm{N1}_{01}$. (From top to bottom and left to right) Initial three-band color image selected at the upper level (i.e., at the fourth resolution level) and the result obtained after convergence of the conjugate gradient. The duplication and result of the gradient conjugate at the finest level of the pyramid, i.e., at full resolution and after the final linear stretching of the three color bands.

the inherent spatial dependencies between neighboring spectral vectors (i.e., modeling the fact that if a given spectral vector belongs to a particular color level class, its surrounding pixels likely belong to the same color class) and, second, the weighted average formula of the nonlocal-means-filtering strategy used in [36], which is very robust to noise. More precisely, in our duplication method, each color level to be interpolated, at site $s$ and at the resolution level $[l-1]$ of $\hat{L}$, is computed as a weighted average of all the color values in the neighborhood of $s$ at level resolution $[l]$ by the following average formula:

$$
L^{[l-1]}(s)=\sum_{t \in \mathcal{N}_{s}} w(s, t) L^{[l]}(s)
$$

where $\mathcal{N}_{s}$ designates a square fixed-size $\left(N_{I}\right)$ neighborhood centered around the pixel $s$. In this interpolation formula, the weights $\{w(s, t)\}_{t}$ depend on the similarity (according to the Euclidean distance) between the spectral vectors of the data cube respectively belonging to pixels $s$ and $t$. Moreover, one must satisfy the usual conditions $0 \leq w(s, t) \leq 1$ and $\sum_{t} w(s, t)=1$ with

$$
w(s, t)=\frac{1}{Z(s)} \exp \left\{-\frac{\|I(s, .)-I(t, .)\|_{2}^{2}}{h}\right\}
$$

where $I$ is the spectral data cube associated to this L-band and $Z(s)$ is the normalizing constant ensuring $\sum_{t} w(s, t)=1$. The parameter $h$ acts as a degree of filtering; it controls the decay of the weights as a function of the Euclidean distance. In our application, we use $h=10$ and $N_{I}=5$.

\section{B. Algorithm}

In order to decrease the computational load of our multiresolution optimization procedure, we only use two levels of resolution in our pyramidal structure (see Fig. 2): the full 


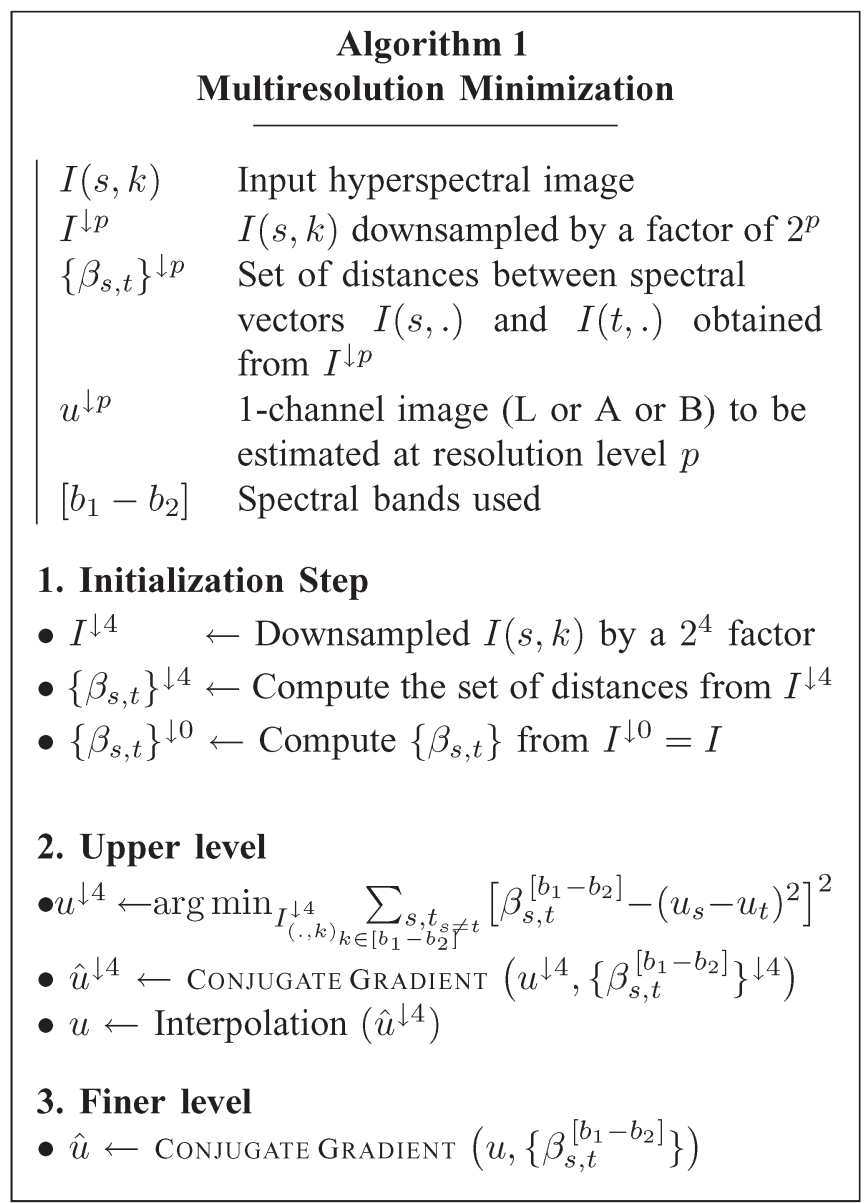

Fig. 3. Algorithm 1: Multiresolution minimization.

resolution and an image 16 times smaller, i.e., corresponding to the fourth upper level of a classical data pyramidal structure (each image is assumed to be toroidal, i.e., periodically repeated). At this lower resolution level, we consider that each node (or pixel) is connected with all other pixels located within a square neighborhood window of fixed-size $N_{s}=30$ pixels centered around the pixel. This allows us to consider nearly a complete graph for an image of size $512 \times 614$, such as an AVIRIS hyperspectral image (once downsampled by a factor 16). At the finest resolution level $(l=0)$, we consider the same square neighborhood window but with $10 \%$ of connections regularly spaced between the pixels located within the neighborhood window. We initialize the lower (or $r=4$ th upper) level of the pyramid (using the strategy mentioned in Section III-A) with the downsampled image candidate $\hat{L}^{[l]}$ which ensures (among the bands between the interval $\left[k_{0}-k_{1}\right]$ ) the minimal energy of the Gibbs energy function of our fusion model. We use a fixed number of iterations for the conjugate gradient at each level of resolution or a stop criterion if the stability of the solution is met. We provide details of our optimization strategy in Algorithm 1 (Fig. 3).

\section{Quantitative Metrics of Visualization Fidelity}

To evaluate the reliability of our algorithm, we will use in the following the correlation-based metric proposed in [10] and [19] and the separability-of-features metric proposed in [19].

\section{A. Preservation of Distances}

This correlation metric is simply the correlation of the Euclidean distance between each pairwise spectral vectors in the hyperspectral image (let $X$ be this vector) and their corresponding (pairwise) Euclidean distances (color difference) in the perceptual LAB color space (let $Y$ be this vector). The correlation $\rho$ can then be estimated by

$$
\rho=\frac{X^{t} Y /|X|-\bar{X} \bar{Y}}{\operatorname{std}(X) \cdot \operatorname{std}(Y)}
$$

where $X^{t},|X|, \bar{X}$, and $\operatorname{std}(X)$ denote the transpose, cardinality, mean, and standard deviation of $X$, respectively. In practice, we consider a subsampling of pairs of pixels in the image (i.e., all pairs with horizontal or vertical (possible) displacements of $2^{p}$ pixels for $p \leq 9$ ). In the absence of perceptual error and, thus, with no loss of information in this dimensionality reduction problem, the ideal correlation metric is one.

It is worth noting that, in the absence of noise (or loss of information due to the dimensionality reduction model), a maximum of this correlation criterion corresponds to the global minima of our energy function [see (1)] (since, if all pairs of pixels preserve their perceptual distance, our energy function equals zero and the correlation metric or $\rho$ function is one). Moreover, the local optimization of these two functions ( $\rho$ and energy function $E$ ) minimizes, in fact, the same criterion, since a small decrease of the correlation factor implies that a certain number of pairwise spectral vectors no longer correspond to their Euclidean distance which necessarily implies an increase in our energy function [(1)]. Let us finally note that our energy function [see (1)] appears more easy to optimize. In addition, a simple analytical expression of the derivative (used in the gradient-based minimization procedure) can be easily found in this case (see Algorithm 2, shown in Fig. 4).

\section{B. Separability of Features}

In order to also measure if there is enough contrast in the final color image, a metric proposed by Cui et al. [19] is able to measure how well the pixels are mapped to distinguishable colors. This criterion has been defined as being simply the average distance between each pair of pixels in perceptual color space (it should be as large as possible): $\delta=|Y|_{1} /|Y|$, where $|Y|_{1}$ and $|Y|$ denote the L1 norm and the cardinality of vector $Y$, respectively.

\section{EXPERIMENTAL RESUlTS}

\section{A. Setup}

In all the experiments, we have thus considered the following setup. For the preprocessing model, we exclude the spectral bands containing mainly noise. To this end, we compute a rough estimation of the signal-to-noise ratio (SNR) of each band by considering roughly the additive noise as the difference between the original image and this image three times filtered by a $3 \times 3$ classical Gaussian filter. We remove bands for which $S N R<S N R_{\min }=10$. If this procedure removes more than 


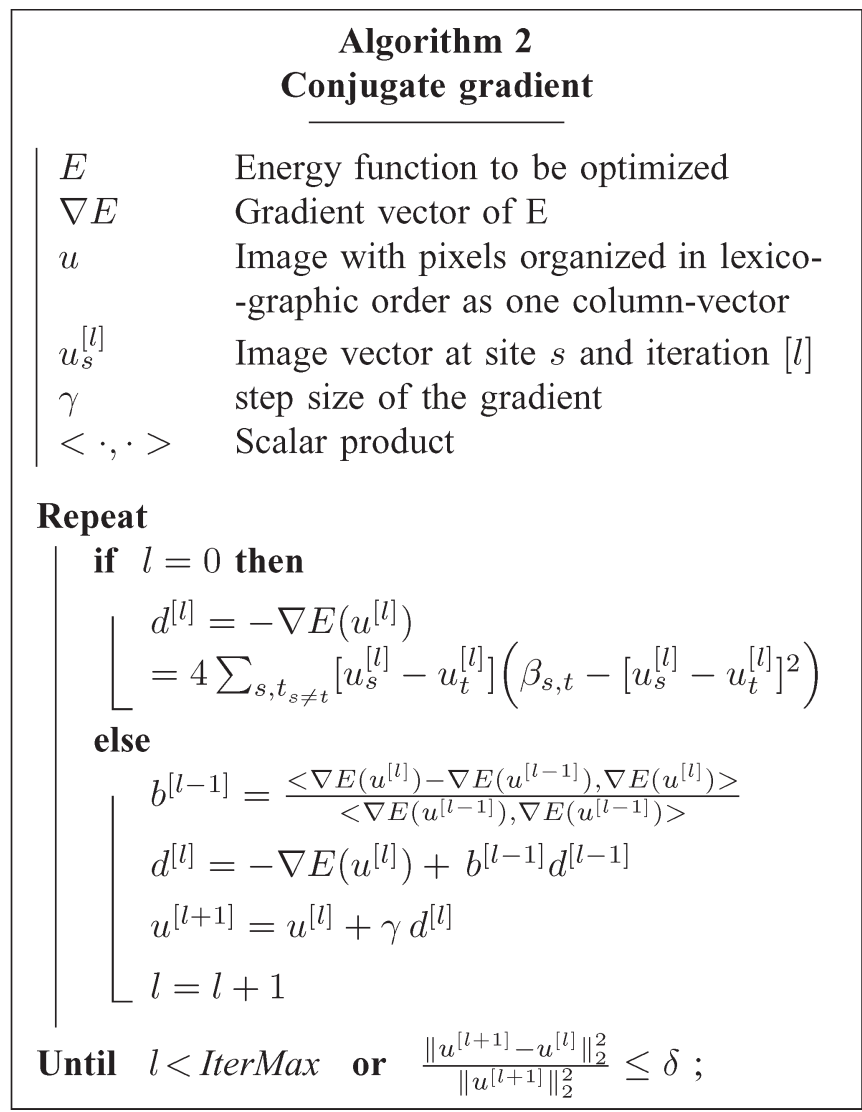

Fig. 4. Algorithm 2: Conjugate gradient.

a third of $K$ initial bands, $S N R_{\min }$ is divided by 1.5 . For the classical AVIRIS LUNAR-LAKE01 image, this preprocessing step removes between six (LUNAR-LAKE ${ }_{02}$ ) and eight bands LUNAR-LAKE $\left.{ }_{01}\right)^{2}{ }^{2}$

For the fusion model, the squared Euclidean distance (divided by the number of considered spectral bands) has been chosen for the computation of $\beta$ [i.e., for the distance between two spectral vectors; see (1)]. For the algorithm, as we have noted, we use two levels of pyramid: the full and the fourth level of resolution with a square neighborhood window of fixed size $N_{s}=30$ pixels, totally connected on the upper level and with $5 \%$ of connections at full resolution level. We have used 40 iterations for the conjugate gradient or less if the stability criterion of the solution is met (see Algorithm 2). For the conjugate gradient, the step size is fixed to $10^{-4}$ and adaptively decreased by a factor of two if the energy to be minimized increases. For the interpolation method summarized in Section III-A, we use $h=12$ and $N_{I}=5$. For the final visualization, we use a final linear stretching (also called "linear fusion of images"

\footnotetext{
${ }^{2}$ Let us note that a similar automatic bad band removal strategy but based on the inter-correlation measure (using the fact that the noisy hyperspectral bands lack structure) has also been proposed in [16] and [17]. Let us note that such a strategy can be also done manually (by visual inspection) or can be determined [10] by the publicly available signal-to-noise curve (due to the measurement equipment) from the 1997 AVIRIS calibration given in [37, Fig. 19]. In our case, our automatic band removal strategy result is very close to that from manual selection and the selection proposed in [10] since the selected bands to be removed by our method [for LUNAR-LAKE ${ }_{01}$, bands 162-168 (1893-1948 $\mathrm{nm})$ and $222(2486 \mathrm{~nm})$ and, for LUNAR-LAKE ${ }_{02}$, bands 161-165 and 222] correspond also to the lowest signal-to-noise bands.
}

\section{Algorithm 3 \\ Final LAB $\rightarrow$ RGB conversion}

1. Linear stretch $L \in[0: 100]$

Linear stretch $A, B \in[-60: 80]$

2. $\mathrm{LAB} \rightarrow \mathrm{RGB}$ color space conversion

$\triangleright$ convert $\mathrm{LAB} \rightarrow \mathrm{XYZ}$

$\triangleright$ convert $\mathrm{XYZ} \rightarrow \mathrm{RGB}$ [38]

$$
\left(\begin{array}{l}
R_{s} \\
G_{s} \\
B_{s}
\end{array}\right)=\left(\begin{array}{ccc}
3.2410 & -1.5374 & -0.4986 \\
-0.9692 & 1.8760 & 0.0416 \\
0.0556 & -0.2040 & 1.0570
\end{array}\right)\left(\begin{array}{l}
X \\
Y \\
Z
\end{array}\right)
$$

sRGB gamma correction

$$
R^{\prime}= \begin{cases}12.92 R_{s} & \text { if } R_{s} \leq 0.00304 \\ 1.055 R_{s}^{(1.0 / 2.4)}-0.055 & \text { otherwise } \\ \text { Similarly for } G^{\prime} \text { and } B^{\prime} . \text { Finally, } \\ R=255.0 \cdot R^{\prime} \\ G=255.0 \cdot G^{\prime} \\ B=255.0 \cdot B^{\prime}\end{cases}
$$

3. Negative color pixels are set to 0 Pixels greater than 255 are set to 255

4. Linear stretch $R, G, B \in[0: 255]$

Fig. 5. Algorithm 3: Final LAB $\rightarrow$ RGB conversion.

in [11]) of the LAB color values, such as $L \in[0: 100]$, and $A, B \in[-60: 80]$ in order to ensure that a very weak minority of pixels are outside the RGB color space. Pixels that are outside the RGB color space, i.e., negative pixels are set to 0 and those that are greater than 255 are set to 255 . The final RGB bands are then stretched between [0:255] (see Algorithm 3 and [38]). Since the image has been (slightly) changed due to this (eventual) saturation, the correlation metric (preservation of distances) is estimated from the LAB conversion of this latter (stretched) RGB image.

\section{B. Hyperspectral Image Used}

The hyperspectral data chosen for the experimental results of our visualization technique are from the National Aeronautics and Space Administration Jet Propulsion Laboratory AVIRIS system [37], which captures 224 spectral bands, ranging from 400 to $2500 \mathrm{~nm}$ with a 1995 AVIRIS. The image size is usually around $512 \times 614$ pixels. We have used the reflectance data, which are atmospherically corrected to compensate for absorption and the spectrum of the sun. The AVIRIS data are generously available for download online. ${ }^{3}$ We compare results for several images and scenes from the 1997 flight lines of Lunar lake, NV; Cuprite, NV; Jasper Ridge, CA; and Moffet Field, CA.

\section{Quantitative Comparison}

We have replicated the scenario of tests and comparisons used in the evaluation of the state-of-the-art multidimensional

\footnotetext{
${ }^{3} \mathrm{http}: / /$ aviris.jpl.nasa.gov/html/aviris.freedata.html
} 
TABLE I

COMPARISON OF CORRELATIONS $\rho$

\begin{tabular}{||c||c||c|c|c|c|c||}
\hline \hline & $\mathbf{M}_{4}$ ICD & {$[19]$} & CMF & PCA & PCA $_{2 \%}$ & PCA $_{h}$ \\
\hline \hline Moffett $_{01}$ & $\mathbf{0 . 9 7 5}$ & 0.96 & 0.94 & 0.91 & 0.68 & 0.46 \\
\hline Moffett $_{02}$ & $\mathbf{0 . 9 8 1}$ & 0.96 & 0.79 & 0.92 & 0.51 & 0.35 \\
\hline Moffett $_{03}$ & $\mathbf{0 . 9 8 1}$ & 0.93 & 0.69 & 0.96 & 0.68 & 0.41 \\
\hline Lun.lake $_{01}$ & $\mathbf{0 . 9 8 3}$ & 0.95 & 0.82 & 0.92 & 0.53 & 0.21 \\
\hline Lun.lake $_{02}$ & $\mathbf{0 . 9 6 0}$ & 0.84 & 0.81 & 0.95 & 0.37 & 0.27 \\
\hline Cuprite $_{01}$ & $\mathbf{0 . 9 7 5}$ & 0.90 & 0.87 & 0.91 & 0.55 & 0.32 \\
\hline Cuprite $_{02}$ & $\mathbf{0 . 9 7 8}$ & 0.91 & 0.88 & 0.95 & 0.43 & 0.28 \\
\hline \hline Average & $\mathbf{0 . 9 7 6}$ & 0.92 & 0.83 & 0.93 & 0.54 & 0.33 \\
\hline \hline
\end{tabular}

TABLE II

COMPARISON OF AVERAGE Distances $\delta$

\begin{tabular}{||c||c||c|c|c|c|c||}
\hline \hline & $\mathbf{M}_{4}$ ICD & {$[19]$} & CMF & PCA & PCA $_{2 \%}$ & PCA $_{\mathbf{h}}$ \\
\hline \hline Moffett $_{01}$ & $\mathbf{1 0 . 3}$ & 38 & 16 & 13 & 50 & 81 \\
\hline Moffett $_{02}$ & $\mathbf{1 1 . 1}$ & 54 & 31 & 12 & 48 & 77 \\
\hline Moffett $_{03}$ & $\mathbf{8 . 1}$ & 25 & 30 & 10 & 45 & 76 \\
\hline Lun.lake $_{01}$ & $\mathbf{1 2 . 4}$ & 51 & 6 & 11 & 52 & 85 \\
\hline Lun.lake $_{02}$ & $\mathbf{1 4 . 2}$ & 60 & 7 & 16 & 44 & 80 \\
\hline Cuprite $_{01}$ & $\mathbf{1 2 . 5}$ & 52 & 5 & 11 & 48 & 81 \\
\hline Cuprite $_{02}$ & $\mathbf{1 8 . 3}$ & 73 & 8 & 14 & 53 & 81 \\
\hline \hline Average & $\mathbf{1 2 . 4}$ & 50.4 & 14.7 & 12.4 & 48.5 & 80.1 \\
\hline \hline
\end{tabular}

TABLE III

RESULTS FOR SOME AVIRIS HYPERSPECTRAL IMAGES

\begin{tabular}{||c||c|c|c||}
\hline \hline $\mathbf{M}_{4} \mathbf{I C D}$ & Correlation $\rho$ & Contrast $\delta$ & Time (Sec.) \\
\hline \hline Cuprite $_{03}$ & 0.965 & 16.9 & 278 \\
\hline Cuprite $_{04}$ & 0.936 & 7.9 & 279 \\
\hline Jasper $_{01}$ & 0.965 & 11.6 & 287 \\
\hline Jasper $_{02}$ & 0.961 & 7.3 & 289 \\
\hline Jasper $_{03}$ & 0.980 & 11 & 284 \\
\hline Jasper $_{04}$ & 0.975 & 7.9 & 284 \\
\hline Jasper $_{05}$ & 0.973 & 6.9 & 284 \\
\hline \hline
\end{tabular}

imagery color display methods described in [19]. In this scenario, we have to test and evaluate our algorithm (called M4ICD for multiresolution Markov model for multidimensional imagery color display) by measuring the quality of the final color mapping based on the two metrics measuring the preservation of distances $(\rho)$ and the separability of features $(\delta)$ with existing techniques. These techniques are the ones proposed in [19], the color matching function (CMF) [10], the classical PCA method with final linear scaling, the PCA with outlier reduction $\left(\mathrm{PCA}_{2 \%}\right.$ ) (which scales the final bands with $2 \%$ of the pixels at the ends of each channel being saturated in order to enhance the contrast), and, finally, the PCA with histogram equalization $\left(\mathrm{PCA}_{h}\right)$ [19]. Results are shown in Tables I and II. Results on other hyperspectral images are given in Table III for possible eventual comparisons with future multidimensional imagery color display algorithms. Display results with comparisons to other techniques are shown in Figs. 6 and 7. Comparison with existing ground-truth classification is shown in Fig. 8.
The comparison of results shows that $\mathrm{PCA}_{2} \%$ and $\mathrm{PCA}_{h}$ have a good score for the separability of features $(\delta)$, i.e., a good contrast in the final displayed image. Nevertheless, this color contrast enhancement is made to the detriment of the overall correlation score, which indicates that the color values of each channel of the final color image are strongly biased and the resulting image (i.e., its color values) is far less informative than the other visualization techniques.

Our method gives the better score of preservation of distance between all the existing visualization techniques with a contrast value that is comparable to the classical PCA technique. Another important characteristic of our method is that it gives a stable value of preservation of distance and contrast scores for a wide variety of images.

The method proposed by Cui et al. [19] gives a good compromise between the preservation of distance criterion and the contrast value. Nevertheless, the preservation of distance score is lower than our method. This lower score is, in our opinion, due both to the linearity of their method and to their preprocessing step, which first uses a classical PCA to a few bands (20 in their application) and an initial segmentation step using a nonoptimal median-cut algorithm that can result in substantial loss of information. Finally, the computational time of our algorithm is about two times lower than the one proposed in [19].

The experimental results (see Table III) show that the AVIRIS image called CUPRITE ${ }_{04}$ (see Fig. 8) obtains the worst "preservation of distance" score $(\rho=0.936)$ obtained by our method. This score is nevertheless comparable with the average score obtained by the classical PCA and by the algorithm proposed in [19] and better than the score obtained by the other tested algorithms. Nevertheless, our method has also the ability to slightly improve this score by simply increasing its computational cost, more precisely, by increasing the number of iterations used in our multiresolution optimization procedure. Fig. 9 shows how this score is slightly improved by increasing its number of iterations.

\section{Discussion}

Our color display model allows one to obtain well-contrasted and highly detailed images without artifacts (such as noise or blurring artifacts due to the reduction/compression model or spectral ringing artifacts, glints, and anomalous fluorescence hot spots or blocking artifacts that are sometimes inherent to multiresolution procedures). Each image efficiently exploits the overall dynamic range of the color values with a good natural-looking color association and no colors that "pop out" strongly. The different regions can be easily identified, and very fine details are visible; for example, in the last hyperspectral image shown in Fig. 6 (see also the two magnified corresponding regions in Fig. 10), we can easily distinguish the small and rectangular shapes of the buildings with their concrete or shiny metal roofing (each building appears as a square of less than four or six pixel size wide in these two images) with very good edge preservation and resolution. Manmade structures and objects are clearly identifiable in these two images. Other color display results given by our algorithm 

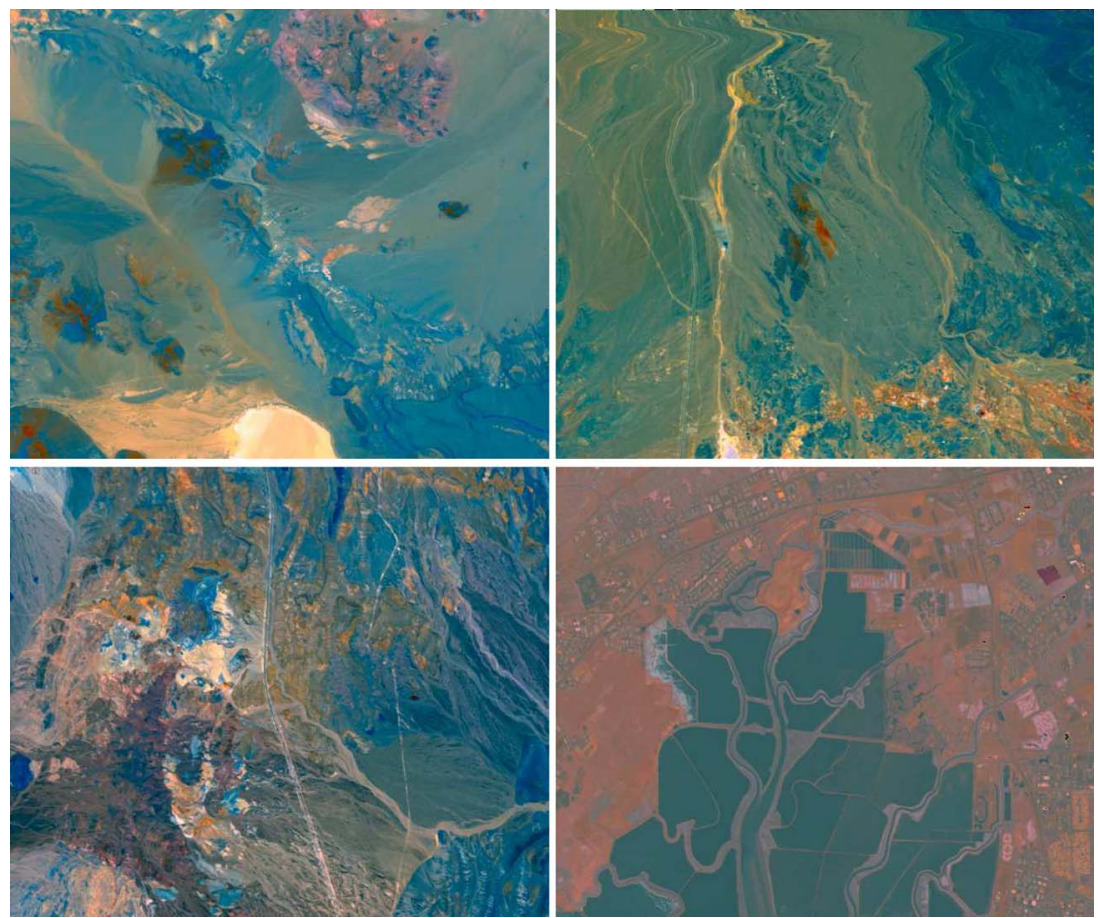

Fig. 6. Different color display results obtained by our M4ICD model. (From top to bottom and left to right) Scenes LUNAR LAKE ${ }_{02}$, CUPRITE ${ }_{01}$, CuPRITE 03 , and MOFFETT $_{02}$. More results are publicly available at the following http address: www.iro.umontreal.ca/ mignotte/ResearchMaterial/m4icd.html.
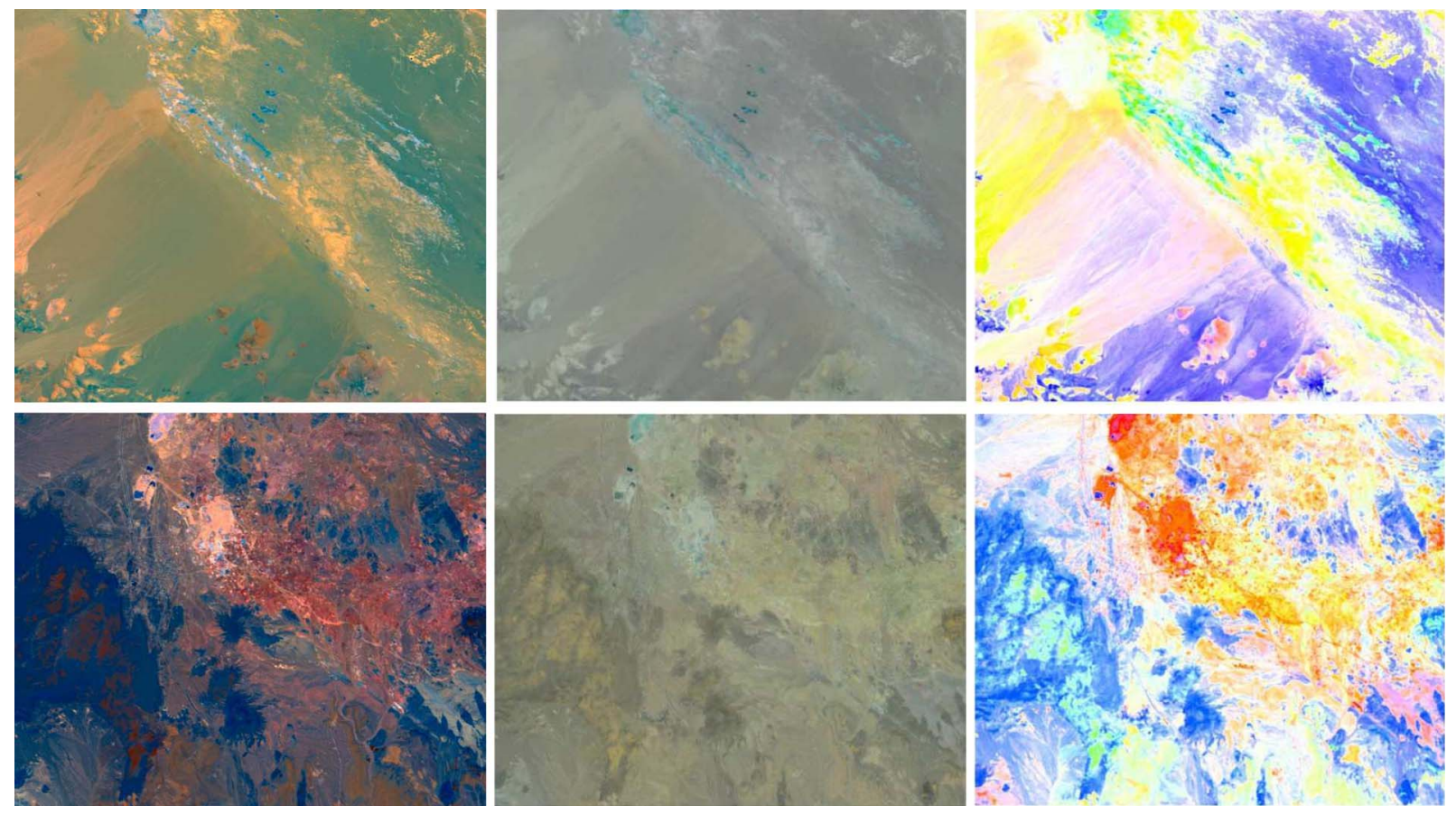

Fig. 7. Comparison of color display results obtained by our M4ICD model and two existing state-of-the-art visualization models: The CMF [10] model and the one proposed in [19] on AVIRIS images LUNAR LAKE 01 and CUPRITE 02 .

are publicly available at the following http address: www. iro.umontreal.ca/ mignotte/ResearchMaterial/m4icd.html.

Let us finally add that we have also experimented a Gauss-Seidel-like approach for the numerical optimization of each equation of the system [see (1); each pixel value is locally updated at each iteration of the Gauss-Seidel-like algorithm by solving a second-degree equation] instead of the conjugategradient method described in Algorithm 2. It turns out that the conjugate-gradient method used herein is much more efficient than the Gauss-Seidel approach (certainly due to the nonlinear equations involved at each iteration of this procedure).

\section{E. Sensitivity to Internal Parameters}

We have also tested the influence of variation of the four internal parameters of our algorithm on the preservation of 

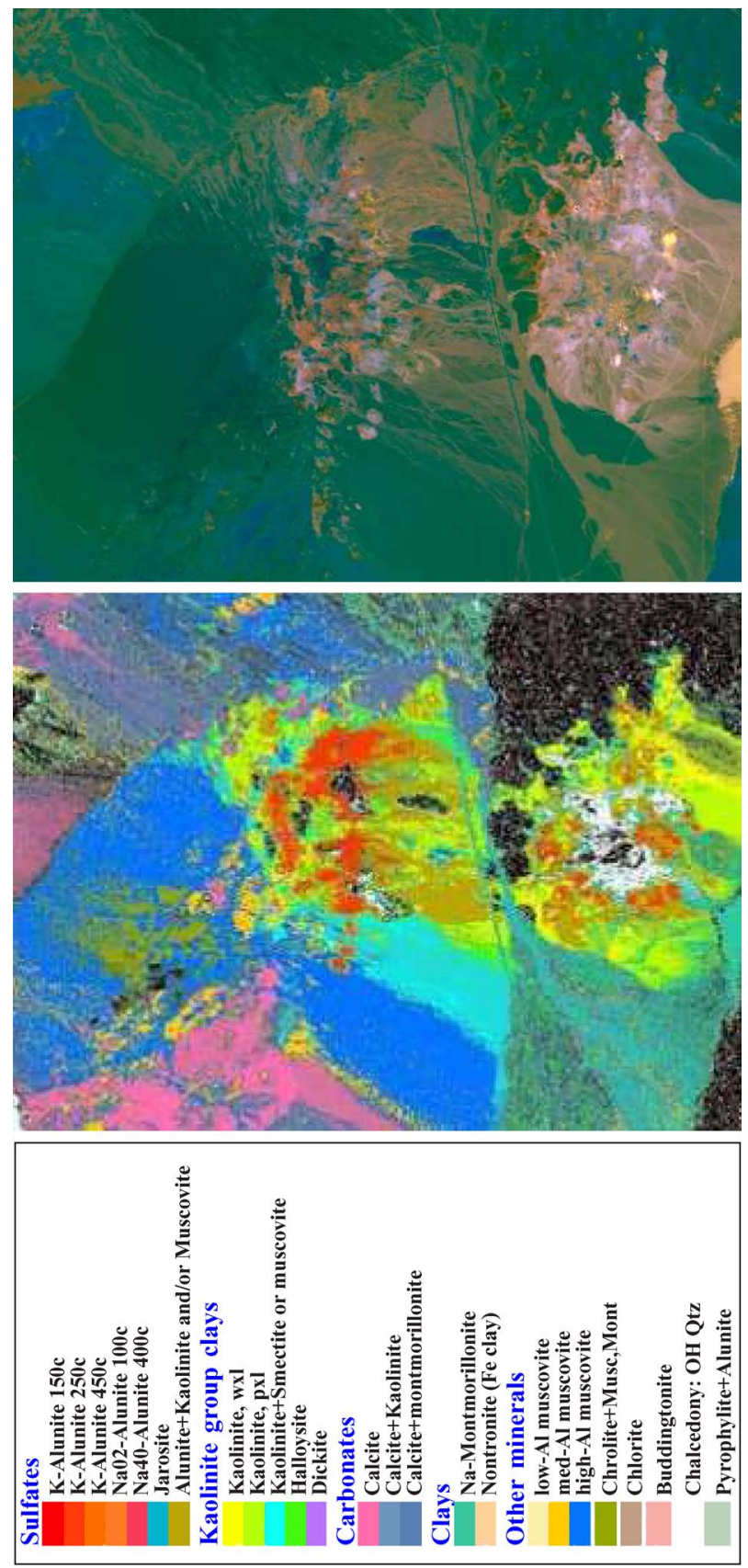

Fig. 8. Comparison of our M4ICD RGB composite display with existing classification ground truths on CUPRITE ${ }_{04}$ AVIRIS image.

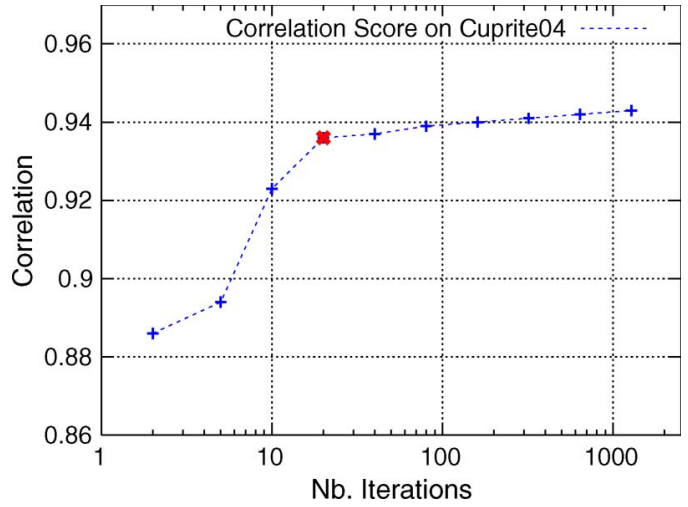

Fig. 9. Evolution of the correlation $\rho$ score as a function of the number of iterations on the CUPRITE ${ }_{04}$ AVIRIS image.
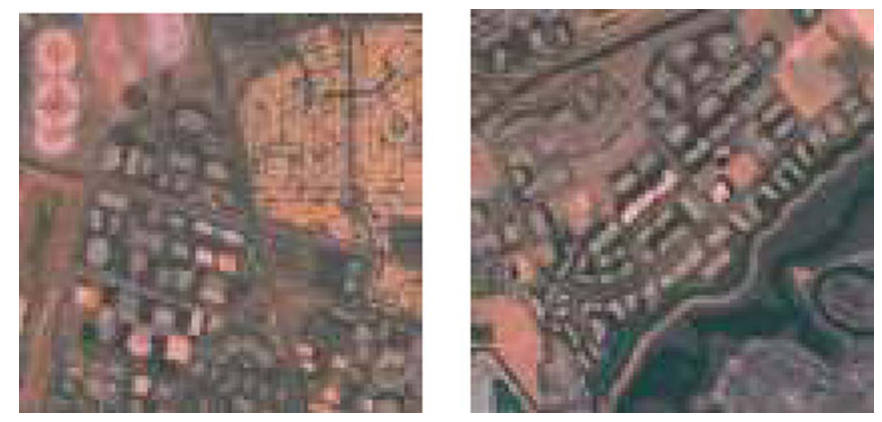

Fig. 10. Magnified regions $(100 \times 100$ pixels $)$ extracted from the last hyperspectral image shown in Fig. 6.

distance and separability-of-feature scores. These parameters are respectively the size of the neighborhood window $N_{s}$, the step size of the conjugate-gradient procedure $\gamma$, the size of the neighborhood window of the interpolation method $N_{I}$, and, finally, the control of the decay of the weights in the nonlocal-means-filtering-based interpolation procedure $h$. Fig. 11 shows the evolution of the preservation of distance score (and separability-of-feature score) for several discrete values of these parameters for the AVIRIS CUPRITE ${ }_{01}$ and CUPRITE 02 scenes. Experiments show that our color display model is not too sensitive to parameters $N_{s}, N_{I}$, and $\gamma$ if $N_{s} \geq 30, N_{I} \geq 5$, and $\gamma \geq 10^{-4}$, respectively (our conjugate-gradient-based optimization procedure remains relatively insensitive to too much high values of the step size $\gamma$ because of our adaptive decreasing schedule which adaptively adjusts and reduces this value if this one is set too high). It also appears that the values of $N_{s}>30$ and $N_{I}>5$ do not allow one to improve the score results but, on the other hand, increase significantly the computational (and memory) requirements. It also appears that $h$ must be in a suitable range, i.e., $h \in[5-30]$, in order to get a reliable interpolation procedure ensuring a good initial guess image (i.e., not too smooth for large values of $h$ and not too noisy for low values of $h$ ).

We have also tested the impact on performances and computational requirement of only one (i.e., without coarser level, $N_{r}=1$, and, thus, a simple full resolution approach) and more than two resolution levels in our multiresolution pyramidal structure (see Table IV). It also appears that more than $N_{r}=2$ resolution levels do not allow one to improve the score results but, on the other hand, increase significantly the computational (and memory) burden. This last experiment means that our multiresolution strategy, including only two levels of resolution (with the coarser level corresponding to the fourth upper level of our pyramid structure), is sufficient to ensure a good initial guess image and allows one to efficiently guide and accelerate the gradient procedure at full resolution toward a good local minima (i.e., very close to the global minima). Without this second coarser level, i.e., with a simple gradient procedure at full resolution $\left(N_{r}=1\right)$, the obtained score remains good, although this score is about 5\% to $10 \%$ less impressive.

\section{F. Algorithm}

The color mapping procedure takes, on average, between 4 and $5 \mathrm{~min}$ (for a $512 \times 614 \times 224$ hyperspectral AVIRIS 


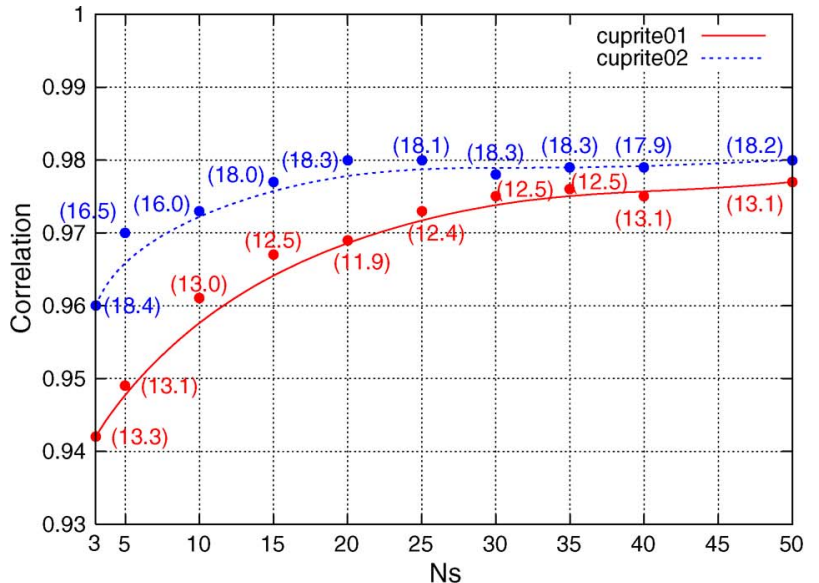

(a)

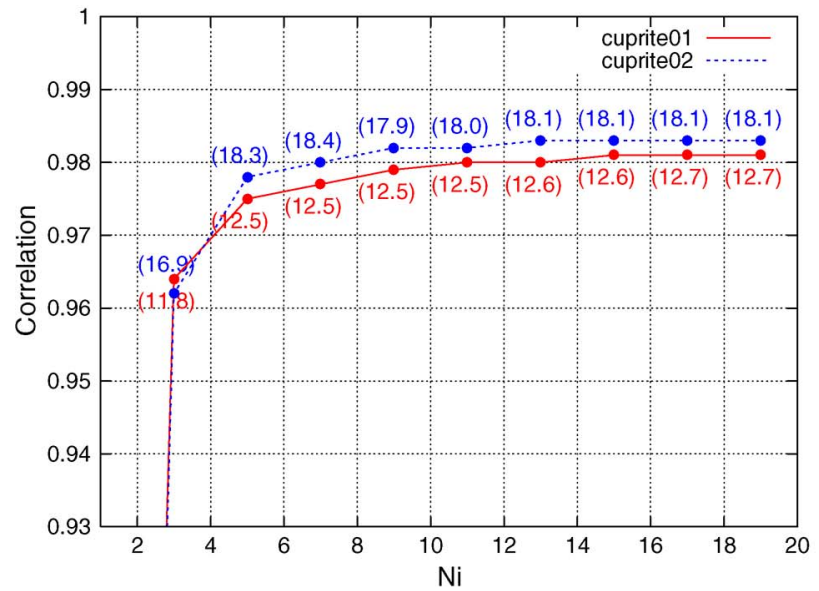

(c)

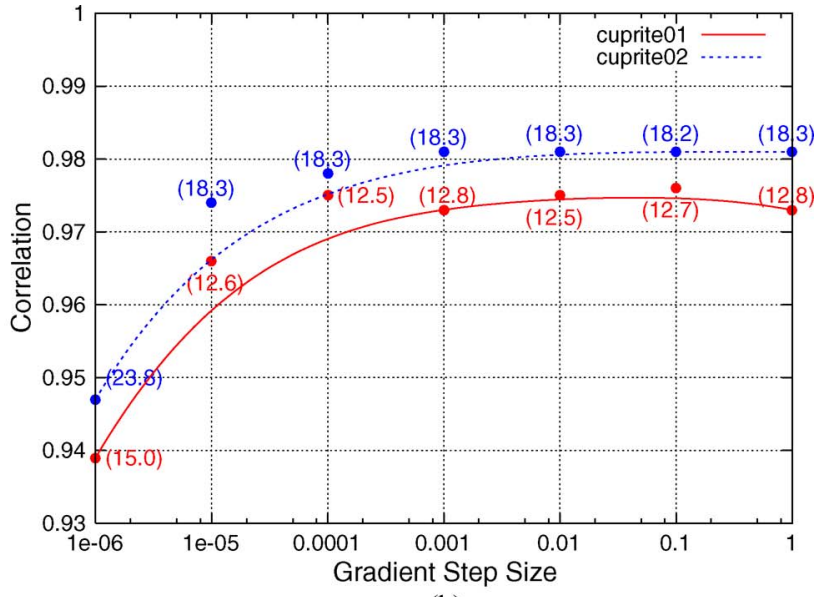

(b)

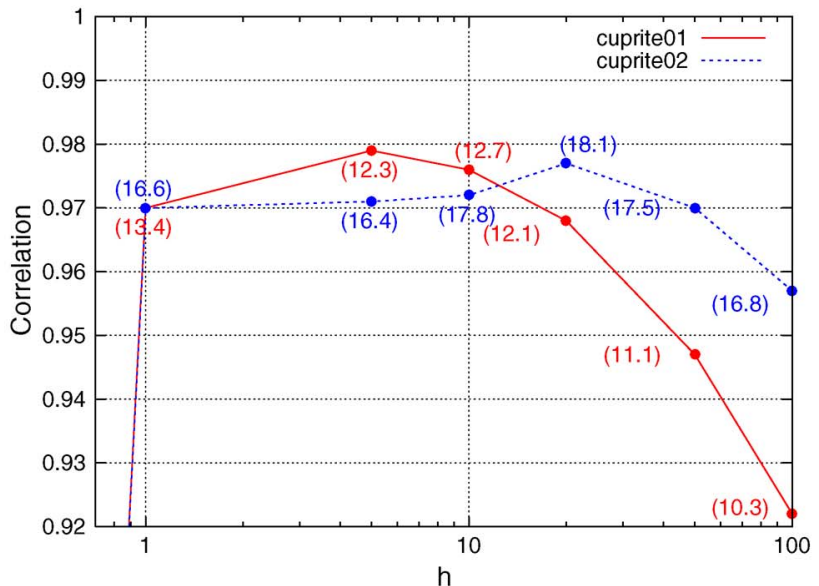

(d)

Fig. 11. Sensitivity to internal parameters. Evolution of the preservation of distance score $\rho$ (and separability-of-features score indicated in []) for CuPRITE 01 and Cuprite ${ }_{02}$ images along (a) $N_{s}$ (with $N_{r}=2$ resolution levels, $\gamma=10^{-3}, N_{I}=5$, and $h=10$ ); (b) $\gamma$ (with $N_{r}=2$ resolution levels, $N_{s}=30$, $N_{I}=5$, and $h=10$ ); (c) $N_{r}=2$ resolution levels and $N_{I}$ (with $N_{s}=30, \gamma=10^{-3}$, and $h=10$ ); and (d) $h$ (with $N_{r}=2$ resolution levels, $\gamma=10^{-3}$, $N_{s}=30$, and $N_{I}=5$ ).

TABLE IV

Impact on Performances (I.E., Preservation of Distances and SeParability-of-Features Scores) and Computational Requirement of Only One (I.E., Without COARser LeVel) AND More Than Two Resolution LeVels in the Multiresolution Pyramidal Structure

\begin{tabular}{|c|c|c|c|c|c|}
\hline & $N_{r}=1$ & $\mathbf{M}_{4}$ ICD & $N_{r}=3$ & $N_{r}=4$ & $N_{r}=5$ \\
\hline Moffett $_{01}$ & $\rho=0.858 \quad \gamma=17.0$ & $\rho=0.975 \quad \gamma=10.3$ & $\rho=0.965 \quad \gamma=9.90$ & $\rho=0.931 \quad \gamma=10.9$ & $\rho=0.936 \quad \gamma=11.1$ \\
\hline Moffett $_{02}$ & $\rho=0.887$ & $\rho=0.981 \quad \gamma=11.1$ & $\rho=0.983$ & $\rho=0.985 \quad \gamma=11.1$ & $\rho=0.987$ \\
\hline Moffett $_{03}$ & $\rho=0.900$ & $\rho=0.981 \quad \gamma=8.10$ & $\rho=0.983$ & $\rho=0.984 \quad \gamma=8.20$ & $\rho=0.983$ \\
\hline Lun.lake $_{01}$ & $\rho=0.973$ & $\rho=0.983 \quad \gamma=12.4$ & $\rho=0.976$ & $\rho=0.979 \quad \gamma=13.9$ & $\rho=0.969$ \\
\hline Lun.lake $_{02}$ & $\rho=0.962$ & $\rho=0.960 \quad \gamma=14.2$ & $\rho=0.963 \quad \gamma=14.6$ & $\rho=0.963 \quad \gamma=14.6$ & $\rho=0.966$ \\
\hline Cuprite $_{01}$ & $\rho=0.978 \quad \gamma=14.5$ & $\rho=0.975 \quad \gamma=12.5$ & $\rho=0.973 \quad \gamma=12.7$ & $\rho=0.972 \quad \gamma=12.8$ & $\rho=0.976 \quad \gamma=13.2$ \\
\hline Cuprite $_{02}$ & $\rho=0.973 \quad \gamma=18.7$ & $\rho=0.978 \quad \gamma=18.3$ & $\rho=0.967 \quad \gamma=16.1$ & $\rho=0.977 \quad \gamma=16.3$ & $\rho=0.981 \quad \gamma=18.2$ \\
\hline Average & $\begin{array}{l}\bar{\rho}=0.933 \quad \bar{\gamma}=14.0 \\
\text { CPU time } \approx 195 \mathrm{sec} .\end{array}$ & $\begin{array}{l}\bar{\rho}=0.976 \quad \bar{\gamma}=12.4 \\
\text { CPU time } \approx 234 \mathrm{sec} .\end{array}$ & $\begin{array}{l}\bar{\rho}=0.973 \quad \bar{\gamma}=14.0 \\
\text { CPU time } \approx 437 \mathrm{sec} .\end{array}$ & $\begin{array}{l}\bar{\rho}=0.970 \quad \bar{\gamma}=12.5 \\
\mathrm{CPU} \text { time } \approx 1080 \mathrm{sec} .\end{array}$ & $\begin{array}{l}\bar{\rho}=0.971 \quad \bar{\gamma}=13.1 \\
\text { CPU time } \approx 5031 \mathrm{sec} .\end{array}$ \\
\hline
\end{tabular}

image) for an AMD Athlon 64 Processor 3500+, $2.2 \mathrm{GHz}$, 4435.67 bogomips, and nonoptimized code running on Linux. Running times in seconds for our methods and for different images are summarized in Table V.

More generally, concerning speed and convergence, our multiresolution optimization scheme gives results close to a stochastic method with a gain of almost two orders of magnitude in terms of speed (which is also confirmed in [21], [35], and
[39]). It must be noted that our Markovian energy minimization can be efficiently implemented by using the parallel abilities of a graphic processor unit [(GPU); embedded on most graphics hardware currently available in the market] and can be greatly accelerated (by a factor of 4 to 200) with a standard NVIDIA GPU (2004), as indicated in [40].

The source code (in C++ language) of our algorithm, called M4ICD (for multiresolution Markov model for 
TABLE V

RUNNING TIME IN SECONDS FOR OUR ALGORITHM

\begin{tabular}{||c||c||}
\hline \hline & $\mathbf{M}_{4}$ ICD \\
\hline \hline Moffett $_{01}$ & 287 \\
\hline Moffett $_{02}$ & 286 \\
\hline Moffett $_{03}$ & 287 \\
\hline Lun.lake $_{01}$ & 295 \\
\hline Lun.lake $_{02}$ & 301 \\
\hline Cuprite $_{01}$ & 280 \\
\hline Cuprite $_{02}$ & 279 \\
\hline \hline
\end{tabular}

multidimensional imagery color display), with the set of resulting three-band color images for each hyperspectral data cube are publicly available at the following http address in order to make possible eventual comparisons with future multidimensional imagery color display algorithms and/or different performance measures: www.iro.umontreal. $\mathrm{ca} /$ mignotte/ResearchMaterial/m4icd.html.

\section{CONCLUSION}

In this paper, we have presented a nonstationary and nonlocal MRF fusion model for the color display of hyperspectral images. This dimensionality reduction model is based on the preservation of spectral distance criterion which ensures a good dimensionality reduction of the spectral data along with meaningful and informative color visualization. In this framework, we have particularly proposed, for the underlying Gibbs energy function related to this Markovian model, an efficient and simple multiresolution optimization strategy based on a conjugate gradient. While being simple to implement, and relatively fast, the proposed procedure allows the nonlinearity of the hyperspectral data to be considered without preprocessing data step and performs competitively among the state-of-theart multidimensional imagery color display methods recently proposed in the literature.

\section{ACKNOWLEDGMENT}

The author would like to thank the anonymous reviewers for their many valuable comments and suggestions that helped improve both the technical content and the presentation quality of this paper.

\section{REFERENCES}

[1] H. Du, H. Qi, X. Wang, and R. Ramanath, "Band selection using independent component analysis for hyperspectral image processing," in Proc. 32nd Appl. Imagery Pattern Recog. Workshop, Washington, DC, Oct. 2003, pp. 93-99.

[2] X. Jia and J. A. Richard, "Segmented principal components transformation for efficient hyperspectral remote-sensing image display and classification," IEEE Trans. Geosci. Remote Sens., vol. 37, no. 1, pp. 538-542, Jan. 1999.

[3] J. Nascimento and J. Dias, "Does independent component analysis play a role in unmixing hyperspectral data?" IEEE Trans. Geosci. Remote Sens., vol. 43, no. 1, pp. 175-187, Jan. 2005.

[4] A. Green, M. Berman, P. Switzer, and M. Craig, "A transformation for ordering multispectral data in terms of image quality with implication for noise removal," IEEE Trans. Geosci. Remote Sens., vol. 26, no. 1, pp. 6574, Jan. 1988.
[5] R. Roger, "A faster way to compute the noise-adjusted principal component transform matrix," IEEE Trans. Geosci. Remote Sens., vol. 32, no. 6, pp. 1194-1196, Jun. 1994.

[6] C.-I Chang and Q. Du, "Interference and noise adjusted principal components analysis," IEEE Trans. Geosci. Remote Sens., vol. 37, no. 9, pp. 2387-2396, Sep. 1999.

[7] P. Switzer and A. A. Green, "Min/max autocorrelation factors for multivariate spatial statistics," Stanford Univ., Stanford, CA, Tech. Rep. 6, 1984.

[8] R. Larsen, A. Nielsen, and K. Conradsen, "Restoration of hyperspectral push-broom scanner data," in Proc. 17th EARSeL Symp. Future Trends Remote Sens., P. Gudmandsen, Ed., Lyngby, Denmark, 1997, pp. 157-162.

[9] V. Tsagaris and V. Anastassopoulos, "Multispectral image fusion for improved RGB representation based on perceptual attributes," Int. J. Remote Sens., vol. 26, no. 15, pp. 3241-3254, Aug. 2005.

[10] N. P. Jacobson and M. R. Gupta, "Design goals and solutions for display of hyperspectral images," IEEE Trans. Geosci. Remote Sens., vol. 43, no. 11, pp. 2684-2692, Nov. 2005.

[11] N. P. Jacobson, M. R. Gupta, and J. Cole, "Linear fusion of image sets for display,” IEEE Trans. Geosci. Remote Sens., vol. 45, no. 10, pp. $3277-$ 3288, Oct. 2007.

[12] S. K. Rogers, T. A. Wilson, and M. Kabrisky, "Perceptual-based image fusion for hyperspectral data," IEEE Trans. Geosci. Remote Sens., vol. 35 , no. 4, pp. 1007-1017, Jul. 1997.

[13] H. Li, B. Manjunath, and S. Mitra, "Multisensor image fusion using the wavelet transform," Graph. Models Image Process., vol. 57, no. 3, pp. 627-640, May 1995.

[14] C. M. Bachmann, T. Ainsworth, and R. Fusina, "Exploiting manifold geometry in hyperspectral imagery," IEEE Trans. Geosci. Remote Sens., vol. 43, no. 3, pp. 441-454, Mar. 2005.

[15] A. Mohan, G. Sapiro, and E. Bosh, "Spatially coherent nonlinear dimensionality reduction and segmentation of hyperspectral images," IEEE Geosci. Remote Sens. Lett., vol. 4, no. 2, pp. 206-210, Apr. 2007.

[16] B. Demir, A. Celebi, and S. Erturk, "A low-complexity approach for the color display of hyperspectral remote-sensing images using onebit-transform-based band selection," IEEE Trans. Geosci. Remote Sens., vol. 47, no. 1, pp. 97-105, Jan. 2009.

[17] S. Cai, Q. Du, and R. J. Moorhead, "Hyperspectral imagery visualization using double layers," IEEE Trans. Geosci. Remote Sens., vol. 45, no. 10, pp. 3028-3036, Oct. 2007.

[18] Q. Du, N. Raksuntorn, S. Cai, and R. Moorhead, "Linear fusion of image sets for display," IEEE Trans. Geosci. Remote Sens., vol. 46, no. 6, pp. 1858-1866, Jun. 2008.

[19] M. Cui, A. Razddan, J. Hu, and P. Wonka, "Interactive hyperspectral image visualization using convex optimization," IEEE Trans. Geosci. Remote Sens., vol. 47, no. 6, pp. 1673-1684, Jun. 2009.

[20] J. M. Duarte-Carvajalino, G. Sapiro, M. Velez-Reyes, and P. E. Castillo, "Multiscale representation and segmentation of hyperspectral imagery using geometric partial differential equations and algebraic multigrid method," IEEE Trans. Geosci. Remote Sens., vol. 46, no. 8, pp. 24182434, Aug. 2008.

[21] A. S. Willsky, "Multiresolution Markov models for signal and image processing," Proc. IEEE, vol. 90, no. 8, pp. 1396-1458, Aug. 2002.

[22] S. Z. Li, Markov Random Field Modeling in Image Analysis, S. P. Company, Ed., 3rd ed. Berlin, Germany: Springer-Verlag, 2009.

[23] S. Geman and D. Geman, "Stochastic relaxation, Gibbs distributions, and the Bayesian restoration of images," IEEE Trans. Pattern Anal. Mach. Intell., vol. PAMI-6, no. 6, pp. 721-741, Nov. 1984.

[24] J. J. Schneider and S. Kirkpatrick, Stochastic Optimization. Berlin, Germany: Springer, 2006.

[25] J. Besag, "On the statistical analysis of dirty pictures," J. R. Stat. Soc. B, vol. 48, no. 3, pp. 259-302, 1986.

[26] Y. Boykov, O. Veksler, and R. Zabih, "Fast approximate energy minimization via graph cuts," IEEE Trans. Pattern Anal. Mach. Intell., vol. 23, no. 11, pp. 1222-1239, Nov. 2001.

[27] V. Kolmogorov and R. Zabih, "What energy functions can be minimized via graph cuts?," IEEE Trans. Pattern Anal. Mach. Intell., vol. 26, no. 2, pp. 147-159, Feb. 2004.

[28] Y. Weiss and W. T. Freeman, "On the optimality of solutions of the maxproduct belief-propagation algorithm in arbitrary graphs," IEEE Trans. Inf. Theory, vol. 47, no. 2, pp. 736-744, Feb. 2001.

[29] P. F. Felzenszwalb and D. P. Huttenlocher, "Efficient belief propagation for early vision," Int. J. Comput. Vis., vol. 70, no. 1, pp. 41-54, Oct. 2006.

[30] M. Wainwright, T. Jaakkola, and A. Willsky, "Map estimation via agreement on trees: Message-passing and linear programming," IEEE Trans. Inf. Theory, vol. 51, no. 11, pp. 3697-3717, Nov. 2005. 
[31] R. Szeliski, R. Zabih, D. Sharstein, O. Veksler, V. Kolmogorov, A. Agarwala, M. Tappen, and C. Rother, "A comparative study of energy minimization methods for Markov random fields with smoothness-based priors," IEEE Trans. Pattern Anal. Mach. Intell., vol. 30, no. 6, pp. 10681080, Jun. 2008

[32] A. Amini, T. Weymouth, and R. Jain, "Using dynamic programing for solving variational problems in vision," IEEE Trans. Pattern Anal. Mach. Intell., vol. 12, no. 9, pp. 855-867, Sep. 1990.

[33] M. Mignotte, "Nonparametric multiscale energy-based model and its application in some imagery problems," IEEE Trans. Pattern Anal. Mach. Intell., vol. 26, no. 2, pp. 184-197, Feb. 2004.

[34] M. Mignotte, C. Collet, P. Perez, and P. Bouthemy, "Sonar image segmentation using a hierarchical MRF model," IEEE Trans. Image Process., vol. 9, no. 7, pp. 1216-1231, Jul. 2000.

[35] M. Mignotte, "A label field fusion Bayesian model and its penalized maximum Rand estimator for image segmentation," IEEE Trans. Image Process., vol. 19, no. 6, pp. 1610-1624, 2010.

[36] A. Buades, B. Coll, and J.-M. Morel, "A review of image denoising algorithms, with a new one," Multiscale Model. Simul. (SIAM Interdisciplinary Journal), vol. 4, no. 2, pp. 490-530, 2005.

[37] G. Vane, R. Green, T. Chrien, H. Enmark, E. Hansen, and W. Porter, "The Airborne Visible/Infrared Imaging Spectrometer (AVIRIS)," Remote Sens. Environ., vol. 44, no. 2/3, pp. 127-143, May/Jun. 1993.

[38] Proposal by Microsoft and Hewlett-Packard, Tech. Rep., IEC 619662.1. Default RGB Colour Space -sRGB M. Stockes, M. Anderson, S. Chandrasekar, and R. Motta, A standard default color space for the Internet -sRGB, Geneva, Switzerland: Int. Electrotech. Commission, Nov. 1996. [Online]. Available: www.color.org/sgrb.html
[39] F. Heitz, P. Pérez, and P. Bouthemy, "Multiscale minimization of global energy functions in some visual recovery problems," CVGIP: Image Understand., vol. 59, no. 1, pp. 125-134, Jan. 1994.

[40] P.-M. Jodoin and M. Mignotte, "Markovian segmentation and parameter estimation on graphics hardware," J. Electron. Imaging, vol. 15, no. 3, pp. 033 015-1-033 015-15, Jul. 2006.

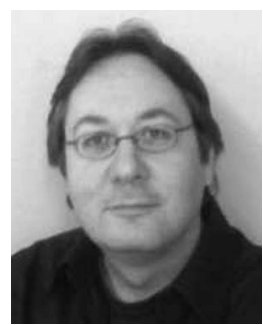

Max Mignotte received the D.E.A. degree in digital signal, image, and speech processing from the Institut Polytechnique de Grenoble, Grenoble, France, in 1993 and the Ph.D. degree in electronics and computer engineering from the Université de Bretagne Occidentale, Brest, France, and the Digital Signal Laboratory, French Naval Academy, France, in 1998.

He was an Institut National de Recherche en Informatique et en Automatique Postdoctoral Fellow with the Département d'Informatique et de Recherche Opérationnelle (DIRO), University of Montreal, Montreal, QC, Canada, from 1998 to 1999. He is currently an Associate Professor (Professeur Agrégé) with the Computer Vision and Geometric Modeling Laboratory, DIRO, University of Montreal. He is also a Member with the Laboratoire de Recherche en Imagerie et Orthopedie, Centre de Recherche du Centre Hospitalier de l'Université de Montréal (CHUM), Hospital NotreDame, and a Researcher with CHUM. His current research interests include statistical methods, Bayesian inference, and hierarchical models for highdimensional inverse problems, such as segmentation, parameter estimation, fusion, deconvolution and restoration, 3-D reconstruction, and shape recognition. 